\title{
End-to-End Resource Allocation in OFDM Based Linear Multi-Hop Networks
}

\author{
Xiaolu Zhang ${ }^{1}$, Wenhua $\mathrm{Jiao}^{2}$, and Meixia $\mathrm{Tao}^{1,3}$, \\ ${ }^{1}$ Dept. of Electrical \& Computer Engineering, National University of Singapore, Singapore 117576 \\ ${ }^{2}$ Bell Lab Research China (BLRC), Alcatel-Lucent, Beijing, China 100080 \\ ${ }^{3}$ Dept. of Electronic Engineering, Shanghai Jiao Tong University, Shanghai, China 200240 \\ Emails: zhangxiaolu@nus.edu.sg; wjiao@alcatel-lucent.com; mxtao@ieee.org
}

\begin{abstract}
We study the end-to-end resource allocation in an OFDM based multi-hop network consisting of a one-dimensional chain of nodes including a source, a destination, and multiple relays. The problem is to maximize the end-to-end average transmission rate under a long-term total power constraint by adapting the transmission power on each subcarrier over each hop and the transmission time used by each hop in every time frame. The solution to the problem is derived by decomposing it into two subproblems: short-term time and power allocation given an arbitrary total power constraint for each channel realization, and total power distribution over all channel realizations. We show that the optimal solution has the following features: the power allocation on subcarriers over each hop has the water-filling structure and a higher water level is given to the hop with relatively poor channel condition; meanwhile, the fraction of transmission time allocated to each hop is adjusted to keep the instantaneous rates over all hops equal. To tradeoff between performance, computational complexity and signalling overhead, three suboptimal resource allocation algorithms are also proposed. Numerical results are illustrated under different network settings and channel environments.
\end{abstract}

\section{INTRODUCTION}

Future wireless systems expect a coverage enhancement and a throughput growth at a low cost. This leads to increasing attentions to the concept of relaying in wireless networks such as next generation cellular networks, broadband wireless metropolitan area networks (WMANs) and wireless local area networks (WLANs) [1], [2]. In recent years, much academic work focuses on ad hoc networks, which can be viewed as generalized relay networks, where each network node may help in relaying each other's data packets. Gupta et al. study the asymptotic bounds of transmission rates with a large number of hops under various network topologies and node capabilities in [3] and [4]. These results, however, do not reveal the actual capacity of a network with a given number of nodes, especially when the number is small. More recently, cooperative communications among the source node and relay nodes in a two-hop scenario is proposed to increase network capacity [5], [6]. The basic idea of cooperative relaying is to achieve diversity through independent channels by controlling

This work is supported in part by National Natural Science Foundation of China under Grant 60572120.

Part of the work of X. Zhang was done when he worked as an intern at BLRC. how the transmitted signals add up at the receiver. Note that cooperative relaying requires precise timing (and possibly phase) synchronization among different nodes which makes it difficult to be integrated in many practical systems. As such, a relay network with a finite number of hops and without synchronous cooperation is of most practical interest.

Relay networks have two possible architectures: a tree of point to multiple points (PMP) architecture and a mesh one. When only a single route is active at any particular time duration, a two-dimensional network can be viewed as multiple one-dimensional chains of nodes. Such a onedimensional chain of nodes, a so-called linear network as in [7], consists of one pair of source and destination nodes and several intermediate relay nodes that are aligned on the straight line between the source and the destination. Transmissions can only occur between two neighboring nodes. In [8], Oyman et al. introduce two different transmission strategies over multiple hops: fixed-rate relaying and rate-adaptive relaying, and showed merits of multi-hop relaying over frequencyflat fading channels. In this work, we consider orthogonal frequency division multiplexing (OFDM) based linear multihop networks over broadband wireless channels. OFDM is an underlying transmission technology to overcome the intersymbol interference imposed by multipath. In fact, the OFDMbased relaying architecture has been accepted by current wireless standards, e.g. IEEE 802.16j. Moreover we adopt time division (TD) for transmissions over different hops so that there is no interference between nodes. Further, time is divided into frames of multiple time slots and the transmission parameters are determined at the beginning of each frame. The transmission parameters include the transmission power on each subcarrier over each hop and the transmission time over each hop, as shown in Fig. 1. When the scheduler knows the global channel state information (CSI), it can perform adaptive relaying. This global information gives a potential for enhancing performance through dynamic resource allocation.

In this paper, we aim to derive the end-to-end maximum average transmission rate in the OFDM-based linear multihop network with adaptive relaying. The problem is first formulated as a max-min problem. We then decompose it into two subproblems: determine the power and time allocation to maximize the end-to-end instantaneous transmission rate under 


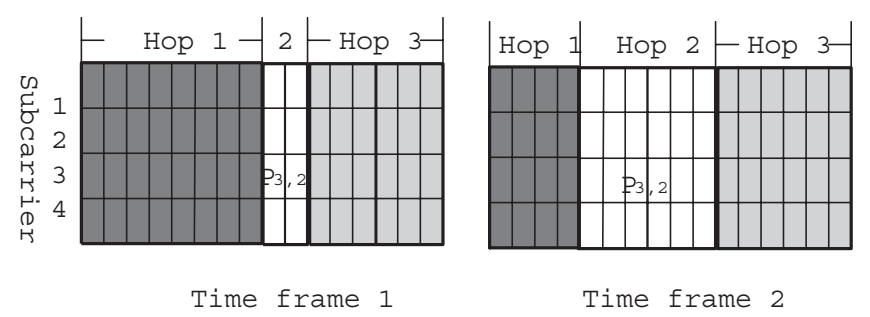

Fig. 1. Illustration of the transmission scheme for an OFDM-based relaying system with 4 subcarriers and 3 hops

a given short-term total power constraint for each channel realization; determine the short-term total power constraint for each channel realization so that the end-to-end average transmission rate is maximized under a long-term total power constraint. Through theoretical analysis, we show that the optimal resource allocation strategy has the following features: the power allocation on the subcarriers at each hop follows the water-filling structure, and the water level varies over time and among different hops. Meanwhile, the fraction of transmission time allocated to each hop is adaptive so that the actual transmission volumes over all hops are equal. The analytical expression for the optimal resource allocation also suggests that the hops with bad channel condition should be given a high water level, and if the channel of any one hop is in deep fade during a certain time frame, the system may be turned off in order to save power. The proposed optimal allocation scheme determines the upper bound of the end-to-end average transmission rate, but also incurs high computational complexity and high signaling overhead. We also propose three suboptimal algorithms with low complexity and reduced overhead. The tradeoff between performance, complexity and overhead is discussed.

The remainder of this paper is organized as follows. The system model is presented in Section II, followed by the problem formulation in Section III. The optimal time sharing fractions and power allocation are derived in Section IV. In Section V, three suboptimal resource allocation algorithms are introduced. Numerical results are given in Section VI. Finally, we conclude this paper in Section VII.

\section{SYSTEM MODEL}

We consider a linear multi-hop wireless network consisting of a source node $R_{0}$, and a destination node $R_{N}$ and $N-1$ relay nodes $R_{n}$ with $n=1, \ldots, N-1$. As shown in Fig. 2, the relays are uniformly aligned on the line from the source to destination. The hop between node $R_{n-1}$ and $R_{n}$ is indexed by $n$, and the set of hops is denoted by $\mathcal{N}$. The transmission time is divided into frames of multiple time slots as shown in Fig. 1. In general, frequency reuse may be possible by allowing multiple hops transmitting at the same time slot. However, due to the interference issue and increased decoding complexity, we will not pursue the frequency reuse in this paper. In each time frame, the message from the source is sequentially relayed at each hop using decode-and-forward scheme [9].

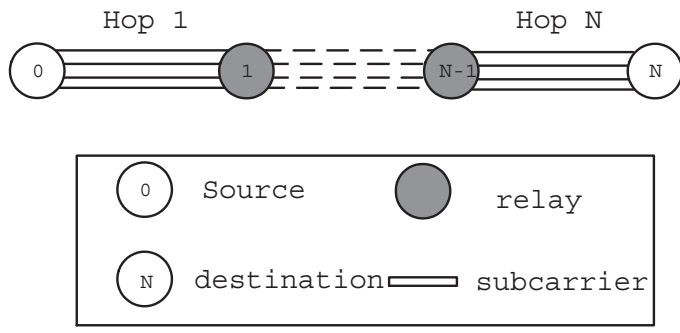

Fig. 2. Illustration of linear multi-hop networks

That is, each relay decodes the message forwarded by the previous node, re-encodes it, and then transmits it to the next relay. Over each hop, OFDM is adopted as the transmission technology at physical layer. It divides the wide-band channel into $K$ narrow-band flat-fading subcarriers indexed by $k$, the set of which is denoted by $\mathcal{K}=\{1,2, \ldots, K\}$. The channel is assumed to be block fading. That is, the channel gain $g_{k, n}$ on subcarrier $k$ over hop $n$ remains invariant during the entire time frame but varies independently from one frame to the other.

Let the transmission power on subcarrier $k$ over hop $n$ be denoted as $p_{k, n}$ and the average total transmission power be constrained by $P$. The fraction of the time frame, $\rho_{n}{ }^{1}$, is used for transmission over hop $n$. The instantaneous transmission rate (Nat/OFDM symbol) in a time frame achieved by subcarrier $k$ over hop $n$ is expressed as

$$
r_{k, n}=\rho_{n} \ln \left(1+\frac{g_{k, n} p_{k, n}}{\Gamma N_{0}}\right),
$$

where $N_{0}$ is the noise power, and $\Gamma$ is the signal-to-noise ratio (SNR) gap for characterizing the difference between the SNR needed to achieve a certain data rate for a practical system and the theoretical limit [10]. For notation brevity, in the remaining part of this paper, we redefine $g_{k, n}$ as the normalized channel gain $g_{k, n}:=g_{k, n} / \Gamma N_{0}$, and let $\mathbf{g}=\left\{g_{k, n}, k \in \mathcal{K}, k \in \mathcal{N}\right\} \in$ $\mathcal{G}$, where $\mathcal{G}$ is the set of all possible normalized channel states. The total instantaneous transmission rate over hop $n$ is denoted as $r_{n}=\sum_{k \in \mathcal{K}} r_{k, n}$. Under the assumption that no data is allowed to accumulate at any of relay nodes, the end-to-end instantaneous transmission rate is equivalent to the minimum of instantaneous transmission rates over $N$ hops [8], [11]. That is, $r=\min _{n \in \mathcal{N}} r_{n}$.

\section{PROBLEM FORMULATION}

In this paper, we consider the scenario where the delay requirement of the application is much longer than the coherence time of the fading channel and, hence, the channel codeword can span multiple time frames. The end-to-end average transmission rate is used to capture the system performance for such delay-tolerant applications. Under the assumption that the channel processes are ergodic, time-average rate is equal to rate averaged over all channel states. In the remainder of this

\footnotetext{
${ }^{1}$ We assume the number of time slots in a time frame is large enough so that $\rho_{n}$ can take arbitrary value between 0 and 1 .
} 
paper, we will use the term average rate for short to distinguish it from the instantaneous rate defined in a particular time frame. We focus on the situations where the current state of the channels over every hop is available at all the transmitters and receivers. Dynamic time and power allocation in response to the channel variations is considered to maximize the endto-end average rate. More specifically, for a given average power constraint $P$, the problem is to determine the optimal transmission power set, $\mathbf{p}(\mathbf{g})=\left\{p_{k, n}, k \in \mathcal{K}, n \in \mathcal{N}\right\}$, and time-sharing fraction set, $\boldsymbol{\rho}(\mathrm{g})=\left\{\rho_{n}, n \in \mathcal{N}\right\}$, as functions of global CSI, $\mathrm{g}=\left\{g_{k, n}, k \in \mathcal{K}, n \in \mathcal{N}\right\}$. This functional optimization problem is formulated as

$$
\begin{aligned}
& \max _{\mathbf{p}(\mathbf{g}), \boldsymbol{\rho}(\mathbf{g})} \quad R(\boldsymbol{\rho}(\mathbf{g}), \mathbf{p}(\mathbf{g})) \\
& \triangleq \mathbb{E}\left[\min _{n \in \mathcal{N}} r_{n}\right] \\
&= \int_{\mathbf{g}}\left[\min _{n \in \mathcal{N}} \rho_{n}(\mathbf{g}) \sum_{k \in \mathcal{K}} c_{k, n}\left(p_{k, n}(\mathbf{g})\right)\right] f(\mathbf{g}) \mathrm{dg}, \\
& \text { s.t. } \mathbb{E}\left(\sum_{k \in \mathcal{K}} \sum_{n \in \mathcal{N}} \rho_{n}(\mathbf{g}) p_{k, n}(\mathbf{g})\right) \leq P \\
& \sum_{n \in \mathcal{N}} \rho_{n}(\mathbf{g})=1
\end{aligned}
$$

where

$$
c_{k, n}(x) \triangleq \ln \left(1+g_{k, n} x\right)
$$

and $f(\mathrm{~g})$ is the joint probability density function (PDF) of the global CSI g.

When $N=1$ and $K=1$, the above problem reduces to characterizing transmission schemes consisting of the direct transmission from source to destination with a single subcarrier. The optimal $R^{*}$ would be the maximum average rate for the single hop transmission in a flat fading channel, and the solution is given by the classic water-filling scheme [12].

\section{Optimal Resource allocation}

In this section, we derive an optimum time and power allocation for $\mathbf{P 1}$. The difficulty in solving the problem arises from the max-min nature of the objective and the co-existence of both long-term and short-term constraints. The long-term power constraint makes it possible to adapt the power not only over hops within one frame but also over frames in the time domain. To make this problem more tractable, we decompose the original problem $\mathbf{P 1}$ into two subproblems.

1) Short-term time and power allocation given an arbitrary total power constraint for each channel realization Consider any given channel state realization $\mathrm{g} \in \mathcal{G}$. Assume that the total power over all $N$ hops assigned to this channel state realization is $p$. The objective is to maximize the end-to-end instantaneous transmission rate. Mathematically, it can be expressed as

$$
\begin{array}{ll}
\text { P2 : } & r(\mathbf{g}, p) \\
& \triangleq \max _{\boldsymbol{\rho}, \mathbf{p}} \min _{n \in \mathcal{N}}\left[\rho_{n} \sum_{k \in \mathcal{K}} c_{k, n}\left(p_{k, n}\right)\right] \\
\text { s.t. } & \sum_{n \in \mathcal{N}} \rho_{n} \sum_{k \in \mathcal{K}} p_{k, n}=p \\
& \sum_{n \in \mathcal{N}} \rho_{n}=1 .
\end{array}
$$

2) Total power distribution $p(\mathbf{g})$ over all channel realizations

The second problem is to determine the instantaneous total power constraint function $p(\mathbf{g})$ over all $N$ hops for each channel realization $\mathrm{g}$ such that the end-toend transmission rate averaged over all channel states is maximized. That is,

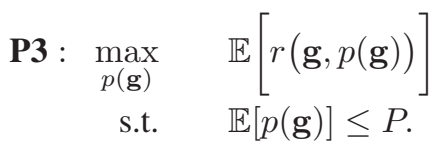

where $r(\mathbf{g}, p(\mathbf{g}))$ is defined in $\mathbf{P 2}$.

Proposition 1: The problem $\mathbf{P 1}$ is equivalent to $\mathbf{P 2}$ when $p$ in (4) is given by the solution $p^{*}(\mathbf{g})$ to $\mathbf{P 3}$.

Proof: See Appendix I.

We solve $\mathbf{P 2}$ and $\mathbf{P 3}$, separately, in the following two subsection.

\section{A. Problem $\boldsymbol{P 2}$}

Problem P2 is a max-min problem with inseparable variables, but it can be transformed into a convex optimization problem,

$$
\begin{aligned}
\text { P4 } \max _{\{r, \boldsymbol{\rho}, \mathbf{s}\}} & r \\
\text { s.t. } & \rho_{n} \sum_{k \in \mathcal{K}} \ln \left(1+\frac{g_{k, n} s_{k, n}}{\rho_{n}}\right) \geq r, \forall n \\
& \sum_{n \in \mathcal{N}} \sum_{k \in \mathcal{K}} s_{k, n}=p \\
& \sum_{n \in \mathcal{N}} \rho_{n}=1
\end{aligned}
$$

where $r$ is a new variable and $s_{k, n} \triangleq \rho_{n} p_{k, n}$. It can be shown that $\rho_{n} \ln \left(1+g_{k, n} s_{k, n} / \rho_{n}\right)$ is concave in both $\rho_{n}$ and $s_{k, n}$. Thus, P4 is a convex optimization problem. The Lagrangian of this problem is given by

$$
\begin{aligned}
J\left(r, \boldsymbol{\rho}, \mathbf{s}, \boldsymbol{\mu}, \lambda_{0}, \nu\right) \\
=r+\sum_{n \in \mathcal{N}} \mu_{n}\left[\rho_{n} \sum_{k \in \mathcal{K}} \ln \left(1+\frac{g_{k, n} s_{k, n}}{\rho_{n}}\right)-r\right]+ \\
\quad \lambda_{0}\left(p-\sum_{n \in \mathcal{N}} \sum_{k \in \mathcal{K}} s_{k, n}\right)+\nu\left(1-\sum_{n \in \mathcal{N}} \rho_{n}\right),
\end{aligned}
$$

where $\boldsymbol{\mu}, \lambda_{0}$ and $\nu$ are all positive and represent the Lagrange multipliers associated with the constraints (8), (9) and (10), respectively. 
Since this problem is convex, the following KKT conditions are necessary and also sufficient for the optimal solution,

$$
\begin{aligned}
\frac{\partial J(\ldots)}{\partial r}= & 1-\sum_{n \in \mathcal{N}} \mu_{n} \\
= & 0, \forall k \in \mathcal{K}, n \in \mathcal{N} \\
\frac{\partial J(\ldots)}{\partial s_{k, n}}= & \frac{\mu_{n} g_{k, n}}{1+g_{k, n} s_{k, n} / \rho_{n}}-\lambda_{0} \\
= & 0, \forall k \in \mathcal{K}, n \in \mathcal{N} \\
\frac{\partial J(\ldots)}{\partial \rho_{n}}= & \mu_{n}\left\{\sum _ { k \in \mathcal { K } } \left[\ln \left(1+\frac{g_{k, n} s_{k, n}}{\rho_{n}}\right)-\right.\right. \\
& \left.\left.\frac{g_{k, n} s_{k, n}}{\rho_{n}+g_{k, n} s_{k, n}}\right]\right\}-\nu=0, \forall n .
\end{aligned}
$$

From (13), we obtain the optimal power allocation as

$$
p_{k, n}^{*}=\frac{s_{k, n}}{\rho_{n}}=\left(\frac{\mu_{n}}{\lambda_{0}}-\frac{1}{g_{k, n}}\right)^{+},
$$

where $(\cdot)^{+}=\max (0, \cdot)$.

Substituting (15) into (14), we obtain the condition for the Lagrange multipliers,

$$
\begin{aligned}
\nu & \triangleq h_{n}\left(\mu_{n}, \lambda_{0}, \mathbf{g}\right) \\
& =\mu_{n} \cdot \sum_{k \in \mathcal{K}}\left\{\left[\ln \left(\frac{\mu_{n} g_{k, n}}{\lambda_{0}}\right)\right]^{+}-\left(1-\frac{\lambda_{0}}{\mu_{n} g_{k, n}}\right)^{+}\right\}
\end{aligned}
$$

for all $n \in \mathcal{N}$. From (16), for a fixed $\lambda_{0}$ and $\nu$, finding the optimal $\boldsymbol{\mu}$ are $N$ independent problem. It can be shown easily that the derivative of the function $h_{n}\left(\mu_{n}, \lambda_{0}, \mathbf{g}\right)$ with respect to $\mu_{n}$ is positive when $\mu_{n} \geq \min _{k}\left\{\lambda_{0} / g_{k, n}\right\}$ and, thus, $h_{n}\left(\mu_{n}, \lambda_{0}, \mathbf{g}\right)$ is monotonically increasing in $\mu_{n}$. The inverse function $h_{n}^{-1}\left(\nu, \lambda_{0}, \mathbf{g}\right)$ thus exists and is an increasing function of $\nu$. As a result, the exact value of $\mu_{n}$ for a given $\nu$ can be obtained numerically using binary search. Substituting $\mu_{n}=h_{n}^{-1}\left(\nu, \lambda_{0}, \mathbf{g}\right)$ into (12), we have

$$
\sum_{n \in \mathcal{N}} h_{n}^{-1}\left(\nu, \lambda_{0}, \mathbf{g}\right)=1
$$

The left side of (17) is monotonically increasing in $\nu$, thus the optimal $\nu$ can also be obtained via binary search from (17). In other words, for a given $\lambda_{0}$, the optimal power allocation can be obtained through two-nested binary search. The outer loop varies the Lagrange multiplier $\nu$ to meet (17). The inner loop searches $\mu_{n}$ at a given value of $\nu$ to satisfy (16). There exists a unique $\lambda_{0}$ such that a positive total power constraint is satisfied.

Proposition 2: The optimal solution to $\mathbf{P 2}$ forces the instantaneous rates over all hops, $r_{n}$ 's, at each time frame to be equal, i.e.,

$$
\rho_{n} \sum_{k \in \mathcal{K}} \ln \left(1+\frac{g_{k, n} s_{k, n}}{\rho_{n}}\right)=\rho_{i} \sum_{k \in \mathcal{K}} \ln \left(1+\frac{g_{k, i} s_{k, i}}{\rho_{i}}\right),
$$

for all $n \neq i$.
Proof: This proposition can be easily obtained by using the KKT conditions in the convex problem P4. An alternative proof is given in Appendix II.

Based on Proposition 2 and the time-sharing constraint (10), each $\rho_{n}$ can be expressed as

$$
\rho_{n}^{*}=\frac{\prod_{i \neq n} \sum_{k \in \mathcal{K}}\left[\ln \left(\frac{\mu_{i} g_{k, i}}{\lambda_{0}}\right)\right]^{+}}{\sum_{i \in \mathcal{N}} \prod_{j \neq i} \sum_{k \in \mathcal{K}}\left[\ln \left(\frac{\mu_{j} g_{k, j}}{\lambda_{0}}\right)\right]^{+}}, \quad \forall n \in \mathcal{N} .
$$

From (18), a more meaningful expression of $\rho_{n}^{*}$ is obtained as

$$
\rho_{n}^{*}=\frac{1}{N} \cdot \frac{\bar{c}}{c_{n}}, \quad \forall n \in \mathcal{N} .
$$

Here, $c_{n}=\sum_{k \in \mathcal{K}}\left[\ln \left(\mu_{n} g_{k, n} / \lambda_{0}\right)\right]^{+}$represents the achievable rate of hop $n$ given power allocation in (15) without time sharing, and $\bar{c}=N / \sum_{n \in \mathcal{N}} \frac{1}{c_{n}}$ is the harmonic mean of $\left\{c_{n}\right\}$ over all the $N$ hops. Now it is clear that the optimal time fraction allocated to each hop is equal to the fraction $1 / N$ scaled by the inverse of the normalized achievable rate on this hop with respect to the harmonic mean of the achievable rates on all hops.

Next, we find out the relation between the maximum endto-end instantaneous transmission rate $r$ and the short-term total transmission power consumption $p$. We assume that $\left\{r^{*}, \boldsymbol{\rho}^{*}, \mathbf{s}^{*}\right\}$ is the solution to P4. Define $\mathcal{K}_{n}$ as the set of active subcarriers $\left(p_{k, n}>0\right)$ over hop $n$, and $k_{n}$ as the size of the set $\mathcal{K}_{n}$. According (15) and (9), the short-term total transmission power can be expressed as

$$
p=\sum_{n \in \mathcal{N}} \rho_{n} \sum_{n \in \mathcal{K}_{n}}\left(\frac{\mu_{n}}{\lambda_{0}}-\frac{1}{g_{k, n}}\right) .
$$

From the above equation, we have

$$
\frac{1}{\lambda_{0}}=\left(p+\sum_{n \in \mathcal{N}} \rho_{n} \sum_{k \in \mathcal{K}_{n}} \frac{1}{g_{k, n}}\right) \frac{1}{\sum_{n \in \mathcal{N}} k_{n} \mu_{n} \rho_{n}} .
$$

We now write the rate $r^{*}$ as a function of $p$ as

$$
\begin{aligned}
r^{*}(p)= & \sum_{n \in \mathcal{N}} \mu_{n}\left[\sum_{k \in \mathcal{K}} r_{k, n}\left(\rho_{n}^{*}, s_{k, n}^{*}\right)\right] \\
= & \sum_{n \in \mathcal{N}} \mu_{n} \rho_{n}^{*}\left[\sum_{k \in \mathcal{K}} \ln \left[\frac{\mu_{n} g_{k, n}}{\lambda_{0}}\right)\right] \\
= & \sum_{n \in \mathcal{N}} \mu_{n} \rho_{n}^{*}\left\{\sum _ { k \in \mathcal { K } } \operatorname { l n } \left[\left(p+\sum_{i \in \mathcal{N}} \rho_{i} \sum_{k \in \mathcal{K}_{i}} \frac{1}{g_{k, i}}\right) .\right.\right. \\
& \left.\left.\frac{\mu_{n} g_{k, n}}{\sum_{i \in \mathcal{N}} k_{i} \mu_{i} \rho_{i}}\right]\right\} .
\end{aligned}
$$

The first equation in (22) rise from Proposition 2. By taking the derivative of (22), and comparing it with (21), it is easy to find that

$$
\frac{d r^{*}(p)}{d p}=\lambda_{0}
$$




\section{B. Problem P3}

With the expression of $r^{*}(p)$ in (22), we now solve $\mathbf{P 3}$ in this subsection. The Lagrangian of Problem (6) is given by

$L\left(p(\mathbf{g}), \boldsymbol{\mu}(\mathbf{g}), \lambda_{0}(\mathbf{g}), \lambda\right)=\int_{\mathbf{g}} l\left(p(\mathbf{g}), \boldsymbol{\mu}(\mathbf{g}), \lambda_{0}(\mathbf{g}), \lambda\right) f(\mathbf{g}) \mathrm{d} \mathbf{g}$,

where

$$
l\left(p(\mathbf{g}), \boldsymbol{\mu}(\mathbf{g}), \lambda_{0}(\mathbf{g}), \lambda\right)=r^{*}(p)-\lambda p(\mathbf{g}) .
$$

According to the generalized KKT necessary condition theorem for variational optimization [13] and using (23), the optimal solution to $\mathbf{P 3}$ satisfies

$$
\frac{\partial l(\ldots)}{\partial p(\mathbf{g})}=\lambda_{0}(\mathbf{g})-\lambda=0
$$

Remarks: One can interpret the Lagrange multiplier $\lambda_{0}$ in (11) as short-term power price for a given channel realization $\mathrm{g}$, and interpret the Lagrange multiplier $\lambda$ in (24) as longterm power price. Equation (25) indicates that the short-term power price is independent of channel condition and equal to the long-term power price.

Combining the results in Sections IV-A and IV-B yields the optimal solution to original problem P1 that satisfies

$$
\begin{gathered}
p_{k, n}^{*}(\mathbf{g})=\left[\frac{\mu_{n}(\mathbf{g})}{\lambda}-\frac{1}{g_{k, n}}\right]^{+}, \forall k \in \mathcal{K}, n \in \mathcal{N}, \\
\rho_{n}^{*}(\mathbf{g})=\frac{\prod_{i \neq n} \sum_{k \in \mathcal{K}}\left[\ln \left(\frac{\mu_{n}(\mathbf{g}) g_{k, n}}{\lambda}\right)\right]^{+}}{\sum_{i \in \mathcal{N}} \prod_{j \neq i} \sum_{k \in \mathcal{K}}\left[\ln \left(\frac{\mu_{j}(\mathbf{g}) g_{k, j}}{\lambda}\right)\right]^{+}}, \quad \forall i \in \mathcal{N} .
\end{gathered}
$$

Thus, in each frame, under the current power price $\lambda$, one can first obtain the optimal transmission power using (26) after two-nested binary search for $\boldsymbol{\mu}$ as described in Section IV$\mathrm{A}$, and then determine the time-sharing fractions using (27) which ensures that the transmission rates $r_{n}$ over all hops are equal. At a larger time scale, we adjust the power price to meet the average power constraint. The adjustment can be implemented using binary search since it can be shown that $\mathbb{E}\left[\sum_{n \in \mathcal{N}} \sum_{k \in \mathcal{K}} \rho_{n}(\mathbf{g}) p_{k, n}(\mathbf{g})\right]$ is monotonically decreasing in the power price.

\section{Properties of the optimal power and time allocation}

It is observed from (26) that the best power allocation has the water-filling structure, where $\mu_{n}(\mathrm{~g}) / \lambda$ can be viewed as the water level.

Proposition 3: If $\sum_{k \in \mathcal{K}_{n}} \frac{1}{g_{k, n}}>\sum_{k \in \mathcal{K}_{i}} \frac{1}{g_{k, i}}$, then we have $\mu_{n} k_{n}>\mu_{i} k_{i}$.

\section{Proof: See Appendix III.}

We treat the total transmission power over hop $n$ as a whole,

$$
p_{n}=\frac{\mu_{n} k_{n}}{\lambda_{0}}-\sum_{k \in \mathcal{K}_{n}} \frac{1}{g_{k, n}} .
$$

where $\mu_{n} k_{n} / \lambda$ is viewed as the water level and $\sum_{k \in \mathcal{K}_{n}} \frac{1}{g_{k, n}}$ is inversely proportional to the harmonic mean of channel gains on the active subcarriers over hop $n$. Proposition 3 suggests that a high water level is given to the hop with small harmonic mean of channel gains to avoid the bottleneck of the whole network it brings. Note that when one of the hops $n$ suffers from a severe fading (the water level is less than $\min _{k} 1 / g_{k, n}$ ) at a particular time frame, equation (27) results in that $\rho_{n}=1$ and $\rho_{i}=0, i \neq n \in \mathcal{N}$, which means the transmission over the whole time frame should be turned off in order to save power for further transmission.

The water-filling power allocation scheme for linear multihop networks is different from the one for multi-user fading broadcast channels in [14] and multi-user uplink channels in [15], in both of which a user uses the same water level over time while different users may have different water levels. This is because the optimal solution for linear multi-hop networks requires that each instantaneous transmission rate over all hops to be equal.

\section{Suboptimal SOLUTiOnS}

In the previous section, we derived the optimal power and time allocation for OFDM based linear multi-hop networks, and refer to it as alg-opt for short. This scheme determines the maximum achievable end-to-end average transmission rate. However, it incurs high computational complexity and large signaling overhead, hence may be infeasible in practice. It is desirable to have suboptimal solutions by imposing some constraints on the network operation. In this section, we develop three suboptimal algorithms, alg1, alg2 and alg3, and discuss their respective computational complexity and signaling overhead.

\section{A. alg1: A solution with a constant water level}

Unlike the optimal scheme, the water level used for power allocation in this suboptimal solution is set to a constant. We express this solution as

$$
\begin{gathered}
p_{k, n}(\mathbf{g})=\left[\frac{1}{\lambda N}-\frac{1}{g_{k, n}}\right]^{+}, \quad \forall k \in \mathcal{K}, n \in \mathcal{N}, \\
\rho_{n}(\mathbf{g})=\frac{\prod_{i \neq n} \sum_{k \in \mathcal{K}}\left[\ln \left(\frac{g_{k, i}}{\lambda N}\right)\right]^{+}}{\sum_{i \in \mathcal{N}} \prod_{j \neq i} \sum_{k \in \mathcal{K}}\left[\ln \left(\frac{g_{k, j}}{\lambda N}\right)\right]^{+}}, \quad \forall n \in \mathcal{N},
\end{gathered}
$$

where $\lambda$ satisfies average power constraint.

We can comprehend alg 1 as follows: first we relax the requirement that transmission rates over all hops equal and maximize the weighted sum of the transmission rates over all hops with weights given by $\left\{\mu_{n}\right\}$. By letting $\mu_{n}=1 / N, \forall n \in$ $\mathcal{N}$ in (26), we can have the expression of $p_{k, n}$ in (28). Then we adjust the time-sharing fractions so that the transmission rates over all hops keep equal. By proposition 3, alg1 approaches to alg-opt when the harmonic mean of the channel gains over active subcarriers, i.e., $k_{n} /\left(\sum_{k=\in \mathcal{K}_{n}} \frac{1}{g_{k, n}}\right)$ of each hop is nearly the same at every time frame. Since the same water level is shared by all subcarriers over all hops and is invariant in the time domain, the computational complexity and signal exchanging overhead can be much reduced. However, a central 
controller is still needed. The difference from the optimal allocation scheme is that there is no need to compute the weight vector $\boldsymbol{\mu}$ at each time frame.

\section{B. alg2: Partially distributed power and time allocation}

The time and power allocation is denoted as

$$
\begin{gathered}
\rho_{n}=\frac{\prod_{i \neq n} \sum_{k \in \mathcal{K}} \mathbb{E}\left[c_{k, i}(P / K)\right]}{\sum_{i \in \mathcal{N}} \prod_{j \neq i} \sum_{k \in \mathcal{K}} \mathbb{E}\left[c_{k, j}(P / K)\right]}, \quad \forall n \in \mathcal{N} . \\
p_{k, n}\left(\mathbf{g}_{n}\right)=\left[\frac{1}{\lambda_{n}}-\frac{1}{g_{k, n}}\right]^{+}, \quad \forall k \in \mathcal{K}, n \in \mathcal{N},
\end{gathered}
$$

where $c_{k, n}(\cdot)$ is given by (3), $\mathbf{g}_{n} \triangleq\left\{g_{k, n}, k \in \mathcal{K}\right\}$ and $\lambda_{n}$ satisfies

$$
\rho_{n} \mathbb{E}_{\mathbf{g}_{n}}\left[\sum_{k \in \mathcal{K}} p_{k, n}\left(\mathbf{g}_{n}\right)\right]=\frac{P}{N} .
$$

$\operatorname{alg} 2$ is a partially distributed algorithm. To avoid centralized management, we require that the average total transmission power is allocated equally to each hop. Since only statistical information is needed to compute the time-sharing fractions and water levels, the signaling overhead can be significantly reduced. Upon receiving the time-sharing fractions, this algorithm performs power allocation across subcarriers in its locality by binary search for $\lambda_{n}$, separately.

\section{C. alg3: Equal resource allocation}

The time and power allocation are denoted as

$$
\begin{aligned}
p_{k, n} & =\frac{P}{K}, \quad \forall k \in \mathcal{K}, n \in \mathcal{N} \\
\rho_{n} & =\frac{1}{N}, \quad \forall n \in \mathcal{N} .
\end{aligned}
$$

Transmission power and time are equally distributed among all subcarriers and hops. It corresponds to the scenario that the transmitters have no channel information. The disadvantage is that any one of hops which suffers from bad channel condition may become the bottleneck of the whole link. This scheme needs no signal exchange and computation.

We list three suboptimal algorithms above in the decreasing order of computational complexity and signaling overhead. Both alg-opt and alg1 need a central controller while alg2 can perform resource allocation at each node side using local information. All of the three suboptimal algorithms are not required to do binary search in each time frame.

According to practical requirements and restrictions, we can choose one of these schemes by finding the optimal tradeoff between performance, complexity and signal exchanging overhead. In the next section, we compare the performance of these algorithms.

\section{NUMERICAL RESULTS}

In this section, we present numerical results of end-to-end average rates under different algorithms. Consider a linear network with $N$ hops. We fix the bandwidth to be $1 \mathrm{MHz}$ and the distance between the source and destination to be $1 \mathrm{~km}$. The relays are equally spaced. In all simulations, the channel over each hop is modelled by Stanford University Interim (SUI)-3 channel model with a central frequency at around 1.9 $\mathrm{GHz}$ to simulate the fixed broadband wireless access channel environments [16]. The SUI-3 channel is a 3-tap channel. The received signal fading on the first tap is characterized by a Ricean distribution with $\mathrm{K}$-factor equal to 1 . The fading on the other two taps follows a Rayleigh distribution. The root-mean-square (rms) delay spread is $0.305 \mu \mathrm{s}$. Then the coherence bandwidth is approximately $65 \mathrm{KHz}$. Hence, the number of subcarriers $K$ should be greater than 15.2 so that the subcarrier bandwidth is small enough to experience the flat fading. Throughout the numerical analysis in this section, we choose $K=16$. The maximal Doppler frequency is set to $0.4 \mathrm{~Hz}$. The path loss model is given by the intermediate path loss condition [17, Category B]:

$$
P L=A+\alpha \lg \left(\frac{d}{d_{0}}\right),
$$

where $d_{0}$ is a reference distance and set to $100 \mathrm{~m}, A=$ $20 \lg \left(4 \pi d_{0} / \lambda\right)$ with $\lambda$ being the wavelength, and $\alpha$ is the pathloss exponent. The SNR gap in (1) is set to $8.2 \mathrm{~dB}$ (which corresponds to a BER requirement of $10^{-5}$ if adaptive QAM modulation is used).

Fig. 3 and Fig. 4 show the end-to-end average transmission rates achieved by using different algorithms with and without shadowing (4dB standard deviation), respectively. From Fig. 3 we observe that the performance gaps between alg2 and alg3 are very small, especially in high power region. Since relays are equally spaced and there is no shadowing, for $\operatorname{alg} 2$, rate over each hop equals on average. Thus, same as alg3, alg2 has uniform time allocation $\rho_{n}=1 / N, \forall n \in \mathcal{N}$. Although alg 2 can vary the transmission power over subcarriers, the impact of power control is limited since the rate is a concave function of the transmission power, especially when the water level is far higher than the inverse of the normalized channel gains. This result in single-hop case was also demonstrated in the work [12]. The improvement of Alg-opt and alg1 over alg 2 is obvious because they adapt the time-sharing fractions according to instantaneous CSI at each time frame and the rate is linear in time-sharing fraction. The similarity in performance of alg1 and alg-opt at the high SNR region indicates that varying water level over time yields a negligible performance gain. Therefore, alg1 and $\operatorname{alg} 3$ are recommended due to their relatively low computational complexity and small signaling overhead. Since different hops suffer independent shadowing, it is more likely that one of all the hops experiences bad channel condition and becomes the bottleneck of the whole link. Thus, the shadowing deteriorates the performance of the algorithm with multiple hops. It also highlights the performance gaps between $a l g 2$ and alg 3 since $a l g 2$ can reduce the impact of the bottleneck by adapting time-sharing fractions according to the channel statistics. On the other hand, alg-opt also does not exhibit a significant rate increase over alg1.

Fig. 5 and Fig. 6 show the the impact of the number of hops at $\alpha=3$ and $\alpha=3.5$, respectively. The optimal algorithm alg-opt is used. It is seen that the optimal number of hops 


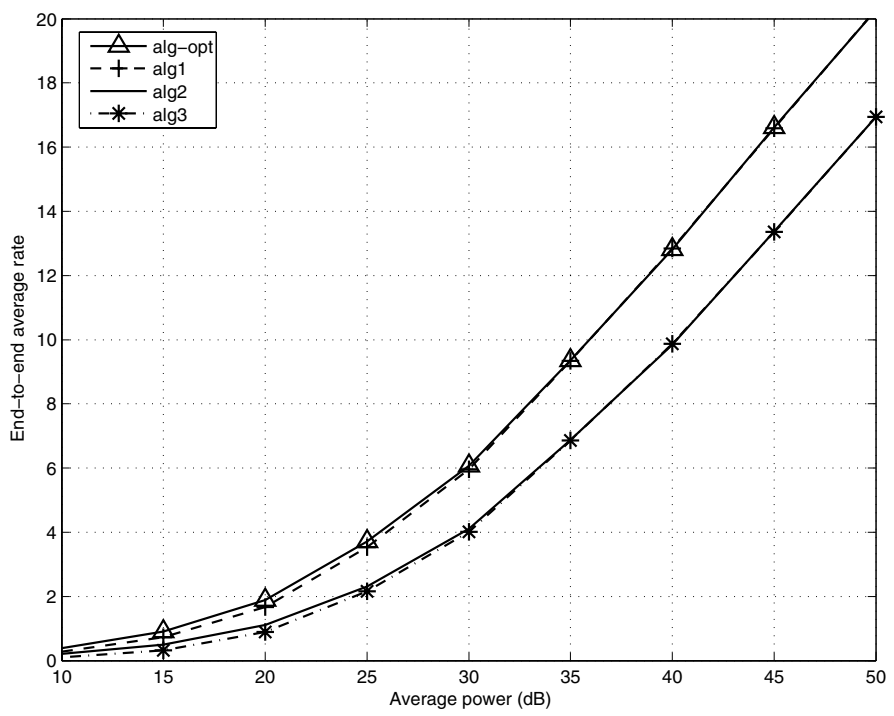

Fig. 3. End-to-end average rate vs. average total transmission power with path loss exponent $\alpha=3.5$, no shadowing and $N=5$

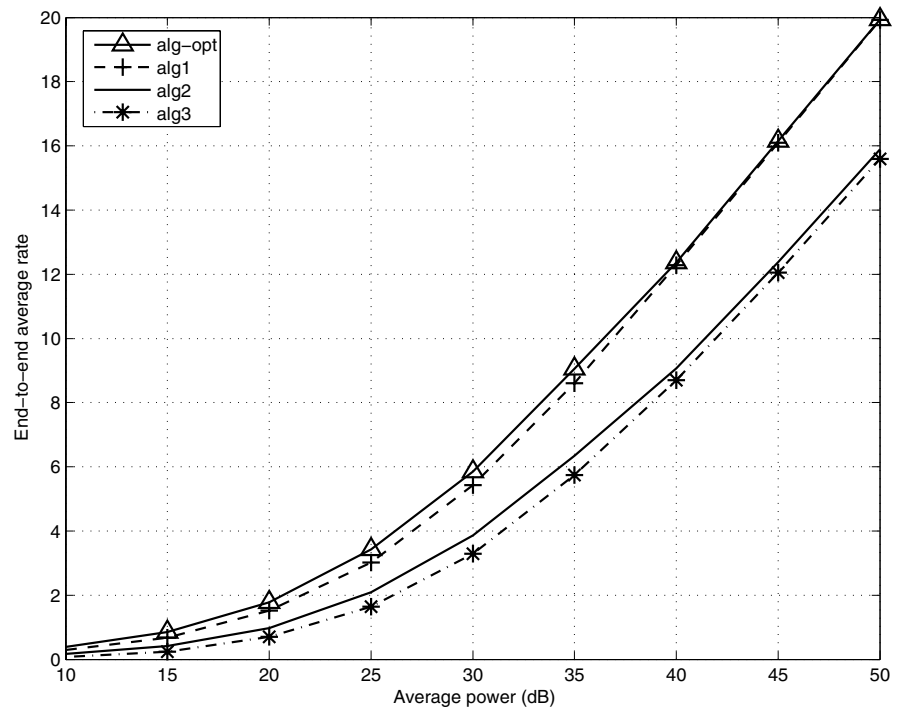

Fig. 4. End-to-end average rate vs. average total transmission power with path loss exponent $\alpha=3.5$, shadowing and $N=5$

deceases as the average total transmission power increases for a fixed path loss exponent. In addition, the intersections of performance curves with different $N$ shift to the left side as the path loss exponent increases.

\section{CONCLUSIONS}

We have obtained the maximum end-to-end average transmission rate for OFDM based linear multi-hop networks and its corresponding optimal power and time allocation. The optimal strategy indicates that the transmission power has the water-filling structure and the hops with relatively bad channel condition are given higher water levels. Further, time-sharing fractions are adaptive so that the transmission rate over each hop equals. We also compare the optimal strategy with three

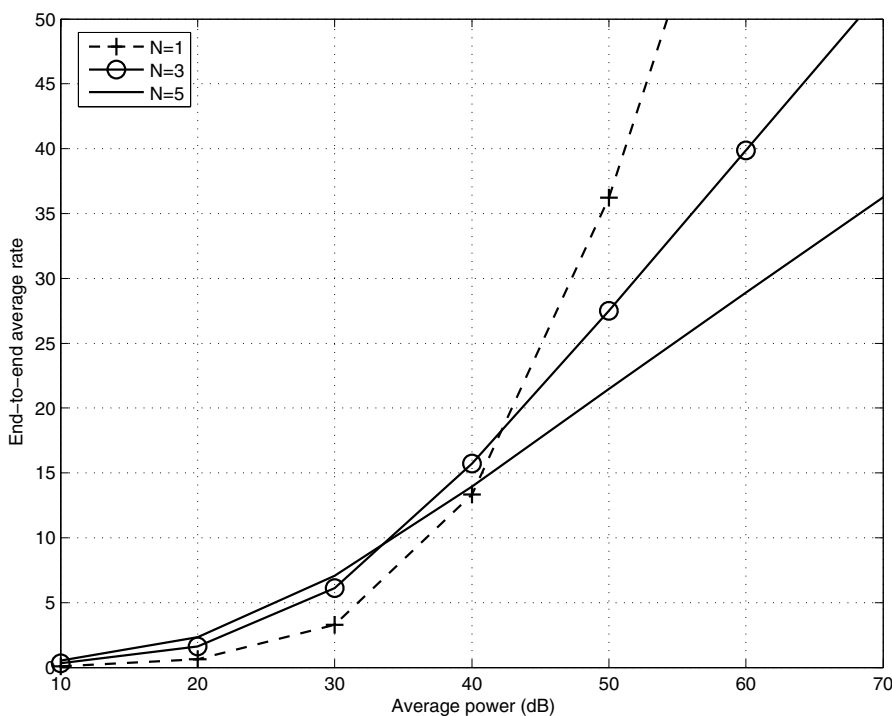

Fig. 5. End-to-end average rate vs. average total transmission power with path loss exponent $\alpha=3$ and no shadowing for alg-opt

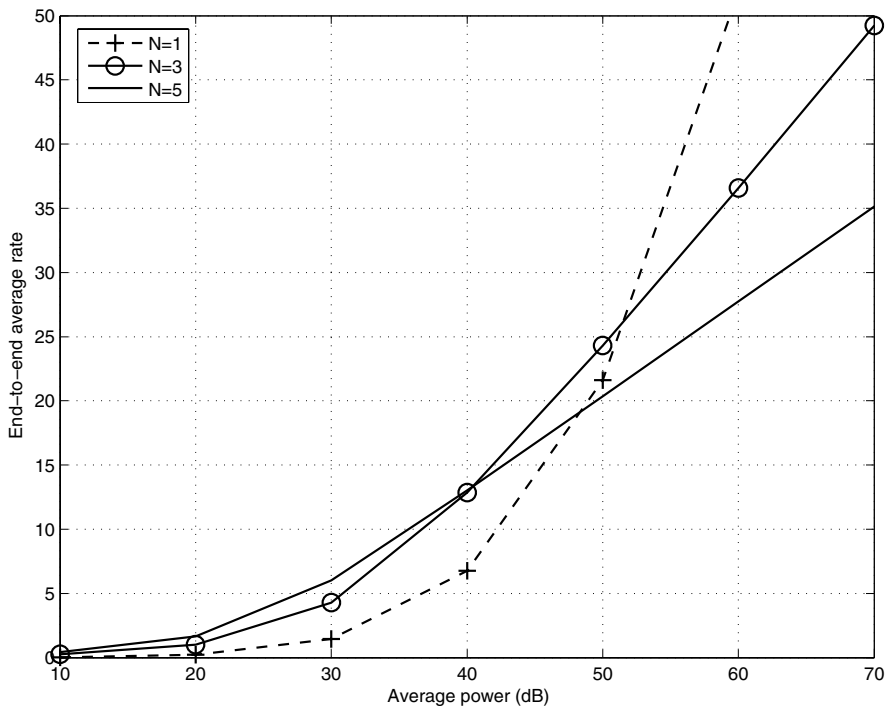

Fig. 6. End-to-end average rate vs. average total transmission power with path loss exponent $\alpha=3.5$ and no shadowing for alg-opt

suboptimal ones which all require lower computational complexity and lower signaling overhead. The numerical findings are that the optimal strategy has a better performance than the equal resource allocation but it does not exhibit a significant rate increase over the suboptimal algorithm with a constant water level.

\section{APPENDIX I}

\section{PROOF OF PROPOSITION 1}

Proof: The equivalence can be proven by contradiction. We assume that $\left(\boldsymbol{\rho}^{\prime}(\mathbf{g}), \mathbf{p}^{\prime}(\mathbf{g})\right)$ is an optimal solution to P1. Let $p^{\prime}(\mathbf{g})=\sum_{n \in \mathcal{N}} \rho_{n}^{\prime}(\mathbf{g}) \sum_{k \in \mathcal{K}} p_{k, n}^{\prime}(\mathbf{g})$ denote the corresponding total power consumption function. Suppose 


$$
\begin{aligned}
f_{0}^{\prime}(p) & =\frac{\mu_{2} k_{2}}{p+\sum_{k \in \mathcal{K}_{2}} \frac{1}{g_{k, 2}}}-\frac{\mu_{1} k_{1}}{p+\sum_{k \in \mathcal{K}_{1}} \frac{1}{g_{k, 1}}} \\
& =\frac{\left(\mu_{2} k_{2}-\mu_{1} k_{1}\right) p+\left(\mu_{2} k_{2} \sum_{k \in \mathcal{K}_{1}} \frac{1}{g_{k, 1}}-\mu_{1} k_{1} \sum_{k \in \mathcal{K}_{2}} \frac{1}{g_{k, 2}}\right)}{\left(p+\sum_{k \in \mathcal{K}_{1}} \frac{1}{g_{k, 1}}\right)\left(p+\sum_{k \in \mathcal{K}_{2}} \frac{1}{g_{k, 2}}\right)} .
\end{aligned}
$$

that $\left(\boldsymbol{\rho}^{\prime}(\mathbf{g}), \mathbf{p}^{\prime}(\mathbf{g})\right)$ is not the optimal solution to $\mathbf{P} \mathbf{2}$ for some channel realization $\mathrm{g} \in \mathcal{G}^{\prime}$ when the short-term power constraint in (4) is given by $p=p^{\prime}(\mathbf{g})$, and $\mathcal{G}^{\prime}$ is the subset of $\mathcal{G}$. We also assume that $\left(\boldsymbol{\rho}^{\prime \prime}(\mathbf{g}), \mathbf{p}^{\prime \prime}(\mathbf{g})\right)$ is the solution to P2 when $\sum_{n \in \mathcal{N}} \rho_{n}^{\prime \prime}(\mathbf{g}) \sum_{k \in \mathcal{K}} p_{k, n}^{\prime \prime}(\mathbf{g})=p^{\prime}(\mathbf{g})$ for all $\mathbf{g} \in \mathcal{G}$. From the above assumptions, we have

$$
\begin{gathered}
\min _{n \in \mathcal{N}}\left[\rho_{n}^{\prime \prime}(\mathbf{g}) \sum_{k \in \mathcal{K}} c_{k, n}\left(p_{k, n}^{\prime \prime}(\mathbf{g})\right)\right]> \\
\min _{n \in \mathcal{N}}\left[\rho_{n}^{\prime}(\mathbf{g}) \sum_{k \in \mathcal{K}} c_{k, n}\left(p_{k, n}^{\prime}(\mathbf{g})\right)\right],
\end{gathered}
$$

for $\mathrm{g} \in \mathcal{G}^{\prime}$. Taking expectation over all $\mathrm{g} \in \mathcal{G}$ for both sides of (32), we have $R\left(\boldsymbol{\rho}^{\prime \prime}(\mathbf{g}), \mathbf{p}^{\prime \prime}(\mathbf{g})\right)>R\left(\boldsymbol{\rho}^{\prime}(\mathbf{g}), \mathbf{p}^{\prime}(\mathbf{g})\right)$ while $\left(\rho^{\prime \prime}(\mathrm{g}), \mathbf{p}^{\prime \prime}(\mathrm{g})\right)$ satisfies all constraints in P1. This result contradicts with the assumption that $\left(\rho^{\prime}(\mathbf{g}), \mathbf{p}^{\prime}(\mathbf{g})\right)$ is an optimal solution to $\mathbf{P 1}$. Therefore, $\left(\boldsymbol{\rho}^{\prime}(\mathbf{g}), \mathbf{p}^{\prime}(\mathbf{g})\right)$ must be the optimal solution to $\mathbf{P 2}$ when the short-term total power constraint is given by $p^{\prime}(\mathrm{g})$. This result also indicates that the end-to-end instantaneous transmission rate can be expressed in terms of the short-term total power as $r\left(\mathrm{~g}, p^{\prime}(\mathrm{g})\right)$. Further, if $p^{\prime}(\mathbf{g})$ is not the optimal solution to $\mathbf{P 3}$, there always exists a $p^{*}(\mathbf{g})\left(\neq p^{\prime}(\mathbf{g})\right)$ such that $\mathbb{E}\left(r\left(p^{*}(\mathbf{g})\right)\right)>\mathbb{E}\left(r\left(p^{\prime}(\mathbf{g})\right)\right)$. To sum up, the optimal solution to $\mathbf{P 1}$ is the same as the one to $\mathbf{P 2}$ where the total power constraint in $\mathbf{P 2}$ is the optimal solution to $\mathbf{P 3}$.

\section{APPENDIX II}

\section{PROOF OF PROPOSITION 2}

Proof: Let $\left(\mathbf{p}^{*}, \boldsymbol{\rho}^{*}\right)$ be the optimal power allocation and time-sharing fraction at time frame $t$. The corresponding instantaneous transmission rate over hop $n$ is denoted as $r_{n}^{*}(\forall n \in \mathcal{N})$. Suppose that for a certain hop $i, r_{i}^{*}>$ $r_{n}^{*}(\forall n \neq i)$. Since $r_{i}$ is a continuous and increasing function of $p_{k, i}(\forall k \in \mathcal{K})$, we can always find a power allocation $p_{k, i}^{\prime}<p_{k, i}^{*}(\forall k \in \mathcal{K})$ such that the corresponding $r_{i}^{\prime}$ satisfies $r_{i}^{*}>r_{i}^{\prime}>r_{n}^{*}(\forall n \neq i)$, while keeping all time-sharing fraction the same $\left(\mathbf{p}^{\prime}=\mathbf{p}^{*}\right)$. That is, we can use a less transmission power to obtain the same instantaneous end-to-end transmission rate at time frame $t$. If we increase the transmission power in time slot $t+1$ by equally allocating the extra power $\sum_{k \in \mathcal{K}}\left(p_{k, i}^{*}-p_{k, i}^{\prime}\right)$ at time slot $t$, the corresponding end-to-end transmission rates at time slot $t+1$ satisfy $r^{\prime}(t+1)>r^{*}(t+1)$. Thus, $\mathbb{E}\left(r^{\prime}\right)>\mathbb{E}\left(r^{*}\right)$. This contradicts the assumption that $\left(\mathbf{p}^{*}, \boldsymbol{\rho}^{*}\right)$ is optimal. Therefore, we have Proposition 2, i.e., $r_{i}^{*}=r_{n}^{*}, \forall i, n \in \mathcal{N}$.

\section{APPENDIX III}

\section{PROOF OF PROPOSITION 3}

Proof: We consider two-hop case, the results of which can be generalized to $N$-hop case. Define

$$
\begin{aligned}
& f_{n}\left(p_{n}\right) \triangleq \max _{\left\{p_{k, n}, k \in \mathcal{K}\right\}} \mu_{n} \sum_{k \in \mathcal{K}} \ln \left(1+g_{k, n} p_{k, n}\right) \\
& \text { s.t. } \sum_{k \in \mathcal{K}} p_{k, n}=p_{n} .
\end{aligned}
$$

Here, $f_{n}(\cdot)$ denotes the weighted total achievable transmission rate on all subcarriers over hop $n$, which is a function of total power on all subcarriers over hop $n, p_{n}$.

We let $p_{n}=x$. According to the basic water-filling theorem [18], the optimal $p_{k, n}$ is denoted as

$$
p_{k, n}=\left(\frac{\mu_{n}}{\lambda_{n}}-\frac{1}{g_{k, n}}\right)^{+} \forall k \in \mathcal{K},
$$

where $\lambda_{n}$ is selected to meet $\sum_{k \in \mathcal{K}} p_{k, n}=x$. Substituting (34) into the above equation, we have

$$
\frac{\mu_{n}}{\lambda_{n}}=\frac{1}{k_{n}}\left(x+\sum_{k \in \mathcal{K}_{n}} \frac{1}{g_{k, n}}\right)
$$

By combining (33), (34) and (35), $f_{n}(x)$ can be denoted as

$$
f_{n}(x)=\mu_{n} \sum_{k \in \mathcal{K}_{n}} \ln \left[\frac{g_{k, n}}{k_{n}}\left(x+\sum_{k \in \mathcal{K}_{n}} \frac{1}{g_{k, n}}\right)\right],
$$

Therefore,

$$
f_{n}^{\prime}(x)=\frac{\mu_{n} k_{n}}{x+\sum_{k \in \mathcal{K}_{n}} \frac{1}{g_{k, n}}}
$$

Define

$$
f_{0}(p)=f_{2}(p)-f_{1}(p),
$$

then we have (36) (see the top of this page).

If $\sum_{k \in \mathcal{K}_{1}} \frac{1}{g_{k, 1}}>\sum_{k \in \mathcal{K}_{2}} \frac{1}{g_{k, 2}}$, then we have $\mu_{1} k_{1}>\mu_{2} k_{2}$. Suppose that $\mu_{1} k_{1} \leq \mu_{2} k_{2}$, the numerator of (36) is nonnegative for any $p>0$. Thus, $\forall p>0, f_{0}^{\prime}(p)>0$. Therefore, $f_{2}(p)>f_{1}(p)$. For $p_{1} \geq 0, p_{2} \geq 0$ and $\rho_{1} p_{1}+\left(1-\rho_{1}\right) p_{2}=p$, we have

$$
\begin{aligned}
\rho_{1} f_{1}\left(p_{1}\right)+\left(1-\rho_{1}\right) f_{2}\left(p_{2}\right) & \leq \rho_{1} f_{2}\left(p_{1}\right)+\left(1-\rho_{1}\right) f_{2}\left(p_{2}\right) \\
& \leq f_{2}(p) .
\end{aligned}
$$

The last inequality is due to the concavity of $f_{2}\left(p_{2}\right)$. This assumption means that all the resource is allocated to hop 2 only. This contradicts the condition that each has the same transmission rate. Therefore, $\mu_{1} k_{1}>\mu_{2} k_{2}$. 


\section{REFERENCES}

[1] R. Bruno, M. Conti, and E. Gregori, "Mesh networks: commodity multihop ad hoc networks," IEEE Commun. Magzine, vol. 43, no. 9, pp. 123-131, 2005.

[2] R. Pabst, B. Walke, D. Schultz, P. Herhold, H. Yanikomeroglu, S. Mukherjee, H. Viswanathan, M. Lott, W. Zirwas, M. Dohler, H. Aghvami, D. Falconer, and G. Fettweis, "Relay-based deployment concepts for wireless and mobile broadband radio," IEEE Comm. Magazine, vol. 42, no. 9, pp. 80-89, 2004.

[3] P. Gupta and P. R. Kumar, "The capacity of wireless networks," IEEE Trans. Inf. Theory, vol. 46, no. 2, pp. 388-404, March 2000.

[4] — " "Toward an information theory of large network: An achievable rate region," IEEE Trans. Inf. Theory, vol. 49, no. 8, pp. 1877-1897, 2003.

[5] J. N. Laneman, D. N. C. Tse, and G. W. Wornell, "Cooperative diversity in wireless networks: efficient protocols and outage behavior," IEEE Trans. Inf. Theory, vol. 50, pp. 3062-3080, 2004.

[6] A. Sendonaris, E. Erkip, and B. Aazhang, "User cooperation diversitypart I: System description," IEEE. Trans. Commun., vol. 51, no. 11, pp. 1927-1938, 2003

[7] M. Sikora, J. N. Laneman, M. Haenggi, J. Daniel J. Costello, and T. E. Fuja, "Bandwidth-and power-efficient routing in linear wireless networks," IEEE Trans. Inf. Theory, vol. 52, pp. 2624-1633, 2006.

[8] O. Oyman, J. N. Laneman, and S. Sandhu, "Multihop relaying for broadband wireless mesh networks: From theory to practice," IEEE Comm. Magazine, pp. 116-122, Nov. 2007.

[9] J. N. Laneman, "Cooperative diversity in wireless networks: Algorithms and architectures," Ph.D. dissertation, Massachusetts Institute of Technology, Cambridge, MA, Aug 2002.

[10] M. Tao, Y. C. Liang, and F. Zhang, "Resource allocation for delay differentiated traffic in multiuser OFDM systems," to appear in IEEE Trans. on Wireless Comm., available at: http://www.ece.nus.edu.sg/stfpage/eletm/publications.htm.

[11] O. Oyman and S. Sandhu, "Non-ergodic power-bandwidth tradeoff in linear multi-hop networks," in Proc. ISIT, July 2006.

[12] A. Goldsmith and P. Varaiya, "Capacity of fading channels with channel side information," IEEE Transa. on Infor. Theory, vol. 43, no. 11, pp. 1986-1992, Nov. 1997.

[13] B. S. Gottfried and J. Weisman, Introduction to optimization theory. Prentice-Hall, 1973.

[14] L. Li and A. Goldsmith, "Capacity and optimal resource allocation for fading broadcast channels: Part I: ergodic capacity," IEEE Trans. Info. Theory, vol. 47, pp. 100-119, 32000.

[15] R. Knopp and P. A. Humblet, "Information capacity and power control in single-cell multiuser communications," in Proc. IEEE Int. Conf. Commun. (ICC), 1995.

[16] V. Erceg, K. Hari, M. Smith, and D. Baum et al, "Channel models for fixed wireless applications," IEEE 802.16.3c-01/29r1, 23 Feb 2001.

[17] V. Erceg, L. Greenstein, S. Tjandra, S. Parkoff, A. Gupta, B. Kulic, A. Julius, and R. Jastrzab, "An empirically based path loss model for wireless channels insuburban environments," IEEE Journal on Selected Areas in Comm., vol. 2, no. 11, pp. 1205-1211, 8-12 Nov. 1999.

[18] R. G. Gallager, Information theroy and reliable communication. New York: Wiley, 1986. 